\title{
Spectral function for excited Dbar meson as the signal of partial restoration of chiral symmetry in the nuclear matter
}

\section{Daiki Suenaga*}

Nagoya University

E-mail: suenagadhken.phys.nagoya-u.ac.jp

\begin{abstract}
We study the masses of $\bar{D}\left(0^{-}\right)$meson and $\bar{D}\left(0^{+}\right)$meson, and spectral function for $\bar{D}_{0}^{*}$ channel in nuclear matter. These mesons are introduced as chiral partner to each other as probes to explore the partial restoration of chiral symmetry in nuclear matter. Our calculations show that the mass difference between $\bar{D}$ meson and $\bar{D}_{0}^{*}$ meson gets small as the baryon number density increases, which reflects the partial restoration of chiral symmetry. In the spectral function for $\bar{D}_{0}^{*}$ meson channel, we find three peaks. First peak corresponds to the resonance of $\bar{D}_{0}^{*}$ meson and this peak is broadened by collisions with nucleons, and peak position shifts to lower energy due to the partial restoration of chiral symmetry. Second peak corresponds to the threshold enhancement, and this peak shifts to higher energy and its height is remarkably enhanced. Third peak is identified as the Landau damping, and this peak grows gradually as the density increases. These modifications are the consequences of partial restoration chiral symmetry in nuclear matter.
\end{abstract}

The 3rd International Symposium on "Quest for the Origin of Particles and the Universe" 5-7 January 2017

Nagoya University, Japan

\footnotetext{
${ }^{*}$ Speaker.
} 


\section{Introduction}

Investigating the chiral symmetry in nuclear matter is one of the most important subject in Quantum Chromodynamics (QCD), since it is expected that chiral symmetry tends to be restored at such environments (see Ref. [W] for a review and references therein). In order to explore chiral symmetry at such extreme region, it is useful to consider a charmed meson such as $\bar{D}(\sim \bar{c} q)$ meson as a probe, since this meson consists of one light quark and one anti-charm quark, so that interactions between $\bar{D}$ meson and nucleons are expected to be rather simple. In this write-up, we provide some main results obtained in our recent work in Ref. [వ]]. In this reference, we studied mass of $\bar{D}\left(0^{-}\right)$meson and $\bar{D}_{0}^{*}\left(0^{+}\right)$meson in nuclear matter and spectral function for $\bar{D}_{0}^{*}$ meson channel, paying attentions to the partial restoration of chiral symmetry in nuclear matter. Our results provides some clues to understand the chiral symmetry in nuclear matter.

\section{Model and calculation}

Before studying the $\bar{D}$ mesons in medium, we need to construct the nuclear matter in order to respect the chiral symmetry. In the present study, we make up the nuclear matter by the linear sigma model as

$$
\begin{aligned}
\mathscr{L}_{\mathrm{LS}}= & \bar{\psi}\left(i \partial+\mu_{B} \gamma^{0}\right) \psi-g \bar{\psi} M_{5} \psi \\
& +\frac{1}{4} \operatorname{Tr}\left[\partial_{\mu} M \partial^{\mu} M^{\dagger}\right]-\frac{m_{0}^{2}}{4} \operatorname{Tr}\left[M M^{\dagger}\right]-\frac{\lambda}{16}\left(\operatorname{Tr}\left[M M^{\dagger}\right]\right)^{2}+\varepsilon \sigma .
\end{aligned}
$$

$\psi$ is the two component nucleon field, and $M$ is the chiral field $M=\sigma+i \tau^{a} \pi^{a}$, where $\sigma$ and $\pi^{a}$ refers the $\sigma$ meson and pion field respectively, and $\tau^{a}$ is the Pauli matrix $(a=1,2,3) . M_{5}$ is defined by $M_{5}=\sigma+i \gamma_{5} \pi^{a} \tau^{a}$. Lagrangian (ㄱ) is invariant under the $S U(2)_{L} \times S U(2)_{R}$ except for the final term in the second line in Lagrangian (L. $(\mathbb{L})$, which is added to give a finite pion mass. $\mu_{B}$ is the baryon number chemical potential. Parameters $g, m_{0}, \lambda$ and $\varepsilon$ are determined in order to reproduce the nucleon mass $m_{N}=939[\mathrm{MeV}]$, pion decay constant $f_{\pi}=92.4[\mathrm{MeV}]$, pion mass $m_{\pi}=138$ $[\mathrm{MeV}]$ and $\pi N$ sigma term $\Sigma_{\pi N}=45[\mathrm{MeV}]$, which reads $g=10.2, \lambda=22.2, m_{0}^{2}=-1.70 \times 10^{5}$ $\left[\mathrm{MeV}^{2}\right]$, and $\varepsilon=1.76 \times 10^{6}\left[\mathrm{MeV}^{3}\right]$.

Lagrangian (L.]) with nucleon one loop provides the effective action

$$
\begin{aligned}
\Gamma[\sigma, \pi]= & -i \operatorname{Tr} \ln \left(i \partial+\mu_{B} \gamma^{0}-g M_{5}\right) \\
& +\int d^{4} x\left(\frac{1}{4} \operatorname{Tr}\left[\partial_{\mu} M \partial^{\mu} M^{\dagger}\right]-\frac{m_{0}^{2}}{4} \operatorname{Tr}\left[M M^{\dagger}\right]-\frac{\lambda}{16}\left(\operatorname{Tr}\left[M M^{\dagger}\right]\right)^{2}+\varepsilon \sigma\right) .
\end{aligned}
$$

Provided that parity invariance in not broken, we can assume a spatially uniform mean field of $\sigma$ meson and pion field: $\langle\sigma\rangle=\sigma_{0}^{*}$ and $\left\langle\pi^{a}\right\rangle=0$, respectively, and can obtained the effective potential $V\left[\sigma_{0}^{*}, 0\right]$ which is defined by $\Gamma\left[\sigma_{0}^{*}, 0\right]=-V\left[\sigma_{0}^{*}, 0\right] \int d^{4} x$. In this configuration, the gap equation $\partial V\left[\sigma_{0}^{*}, 0\right] / \partial \sigma_{0}^{*}=0$ is provided as

$$
\sigma_{0}^{*}\left(m_{0}^{2}+\lambda \sigma_{0}^{* 2}-\varepsilon\right)=-\frac{2 g \sigma_{0}^{*}}{\pi^{2}} \int_{0}^{k_{F}} d|\vec{k}| \frac{|\vec{k}|^{2}}{\sqrt{|\vec{k}|^{2}+m_{N}^{* 2}}}
$$


where $k_{F}$ is the Fermi momentum defined by $\mu_{B}=\sqrt{k_{F}^{2}+m_{N}^{* 2}}$, and $m_{N}^{*}=g \sigma_{0}^{*}$ is the medium modified nucleon mass. Fermi momentum $k_{F}$ is related to the baryon number density $\rho_{B}$ by $\rho_{B}=\left(2 k_{F}^{3}\right) /\left(3 \pi^{2}\right)$. In obtaining $([2.3)$, we have remained the momentum integral which is only dependent on the Fermi momentum $k_{F}$ explicitly. The gap equation in Eq. ([2.3) determines the density dependence of the mean field $\sigma_{0}^{*}$. Furthermore, the effective action (2.2) provides us with the two-point functions of $\sigma$ meson and pion, which is necessary to calculate the one loop corrections to the self-energy of $\bar{D}$ mesons in the nuclear matter.

Next, we construct an effective Lagrangian for $\bar{D}$ mesons interacting with $\sigma$ meson and pion. We start by an effective Lagrangian for $\bar{D}$ mesons which is based on the idea of chiral partner structure, i.e., mass difference between $\bar{D}\left(0^{-}\right)$and $\bar{D}_{0}^{*}\left(0^{+}\right)$mesons, and $\bar{D}^{*}\left(1^{-}\right)$and $\bar{D}_{1}\left(1^{+}\right)$ mesons are driven by the spontaneous breakdown of chiral symmetry [3]. The Lagrangian in the relativistic form which is invariant under the $S U(2)_{S}$ heavy quark spin symmetry and $S U(2)_{L} \times$ $S U(2)_{R}$ chiral symmetry and parity is given by

$$
\begin{aligned}
\mathscr{L}= & \partial_{\mu} \bar{D}_{0}^{*} \partial^{\mu} \bar{D}_{0}^{* \dagger}-m^{2} \bar{D}_{0}^{*} \bar{D}_{0}^{* \dagger}-\partial_{\mu} \bar{D}_{1 v} \partial^{\mu} \bar{D}_{1}^{\dagger v}+\partial_{\mu} \bar{D}_{1 v} \partial^{v} \bar{D}_{1}^{\dagger \mu}+m^{2} \bar{D}_{1 \mu} \bar{D}_{1}^{\dagger \mu} \\
& +\partial_{\mu} \bar{D} \partial^{\mu} \bar{D}^{\dagger}-m^{2} \bar{D} \bar{D}^{\dagger}-\partial_{\mu} \bar{D}_{v}^{*} \partial^{\mu} \bar{D}^{* \dagger \nu}+\partial_{\mu} \bar{D}_{v}^{*} \partial^{v} \bar{D}^{* \dagger \mu}+m^{2} \bar{D}_{\mu}^{*} \bar{D}^{* \dagger \mu} \\
& -\frac{\Delta_{m}}{2 f_{\pi}} m\left[\bar{D}_{0}^{*}\left(M+M^{\dagger}\right) \bar{D}_{0}^{* \dagger}-\bar{D}_{1 \mu}\left(M+M^{\dagger}\right) \bar{D}_{1}^{\dagger \mu}-\bar{D}\left(M+M^{\dagger}\right) \bar{D}^{\dagger}+\bar{D}_{\mu}^{*}\left(M+M^{\dagger}\right) \bar{D}^{* \mu \dagger}\right] \\
& -\frac{\Delta_{m}}{2 f_{\pi}} m\left[\bar{D}_{0}^{*}\left(M-M^{\dagger}\right) \bar{D}^{\dagger}-\bar{D}_{1 \mu}\left(M-M^{\dagger}\right) \bar{D}^{* \dagger \mu}-\bar{D}\left(M-M^{\dagger}\right) \bar{D}_{0}^{* \dagger}+\bar{D}_{\mu}^{*}\left(M-M^{\dagger}\right) \bar{D}_{1}^{\dagger \mu}\right] \\
& -\frac{g_{A}}{2} \frac{m}{f_{\pi}}\left[\bar{D}_{1}^{\mu}\left(\partial_{\mu} M^{\dagger}-\partial_{\mu} M\right) \bar{D}_{0}^{* \dagger}-\bar{D}_{0}^{*}\left(\partial_{\mu} M^{\dagger}-\partial_{\mu} M\right) \bar{D}_{1}^{\dagger \mu}-\frac{1}{m} \varepsilon^{\mu v \rho \sigma} \bar{D}_{1 \mu}\left(\partial_{v} M^{\dagger}-\partial_{v} M\right) i \partial_{\sigma} \bar{D}_{1 \rho}^{\dagger}\right] \\
& +\frac{g_{A}}{2} \frac{m}{f_{\pi}}\left[\bar{D}^{* \mu}\left(\partial_{\mu} M^{\dagger}-\partial_{\mu} M\right) \bar{D}^{\dagger}-\bar{D}\left(\partial_{\mu} M^{\dagger}-\partial_{\mu} M\right) \bar{D}^{* \dagger \mu}-\frac{1}{m} \varepsilon^{\mu v \rho \sigma} \bar{D}_{\mu}^{*}\left(\partial_{v} M^{\dagger}-\partial_{v} M\right) i \partial_{\sigma} \bar{D}_{\rho}^{* \dagger}\right] \\
& +\frac{g_{A}}{2} \frac{m}{f_{\pi}}\left[\bar{D}_{1}^{\mu}\left(\partial_{\mu} M^{\dagger}+\partial_{\mu} M\right) \bar{D}^{\dagger}+\bar{D}\left(\partial_{\mu} M^{\dagger}+\partial_{\mu} M\right) \bar{D}_{1}^{\dagger \mu}\right] \\
& -\frac{g_{A}}{2} \frac{m}{f_{\pi}}\left[\bar{D}_{0}^{*}\left(\partial_{\mu} M^{\dagger}+\partial_{\mu} M\right) \bar{D}^{* \dagger \mu}+\bar{D}^{* \mu}\left(\partial_{\mu} M^{\dagger}+\partial_{\mu} M\right) \bar{D}_{0}^{* \dagger}\right] \\
& -\frac{g_{A}}{2} \frac{1}{f_{\pi}}\left[\varepsilon^{\mu v \rho \sigma} \bar{D}_{1 v}\left(\partial_{\rho} M^{\dagger}+\partial_{\rho} M\right) i \partial_{\sigma} \bar{D}_{\mu}^{* \dagger}+\varepsilon^{\mu v \rho \sigma} \bar{D}_{\mu}^{*}\left(\partial_{\rho} M^{\dagger}+\partial_{\rho} M\right) i \partial_{\sigma} \bar{D}_{1 v}^{\dagger}\right] .
\end{aligned}
$$

In Lagrangian (2.4), $m, \Delta_{m}$ and $g_{A}$ are the parameters which are determined as following. In the vacuum, chiral symmetry is broken by the vacuum expectancy value (VEV) of $\sigma$ field as $\langle\sigma\rangle_{0}=f_{\pi}$, then Lagrangian (2.4) provides $\bar{D}$ and $\bar{D}^{*}$ mesons with the same mass, and $\bar{D}_{0}^{*}$ and $\bar{D}_{1}$ mesons with the same mass due to the $S U(2)_{S}$ heavy quark spin symmetry as $M_{\left(\bar{D}, \bar{D}^{*}\right)}=m-\Delta_{m} / 2$ and $M_{\left(\bar{D}_{0}^{*}, \bar{D}_{1}\right)}=m+\Delta_{m} / 2$. Hence, $m$ is identical to the average mass of $H=\left(\bar{D}, \bar{D}^{*}\right)$ and $G=\left(\bar{D}_{0}^{*}, \bar{D}_{1}\right)$, while $\Delta_{m}$ is identical to the mass difference of them. When we estimate $M_{\left(\bar{D}, \bar{D}^{*}\right)}$ and $M_{\left(\bar{D}_{0}^{*}, \bar{D}_{1}\right)}$ by the spin averaged mass: $M_{\left(\bar{D}, \bar{D}^{*}\right)}=\left(m_{\bar{D}}+3 m_{\bar{D}^{*}}\right) / 4$ and $M_{\left(\bar{D}_{0}^{*}, \bar{D}_{1}\right)}=\left(m_{\bar{D}_{0}^{*}}+3 m_{\bar{D}_{1}}\right) / 4\left(m_{\bar{D}}=1869\right.$ $\mathrm{MeV}, m_{\bar{D}^{*}}=2010 \mathrm{MeV}, m_{\bar{D}_{0}^{*}}=2318 \mathrm{MeV}$ and $m_{\bar{D}_{1}}=2427 \mathrm{MeV}$ are the mass of $\bar{D}, \bar{D}^{*}, \bar{D}_{0}^{*}$ and $\bar{D}_{1}$ mesons in the vacuum respectively), $m$ and $\Delta_{m}$ is determined as $m=2190 \mathrm{MeV}$ and $\Delta_{m}=430$ $\mathrm{MeV}$, respectively. $g_{A}$ is estimated by the decay width of $\Gamma\left[D^{*} \rightarrow D \pi\right]$ as $g_{A}=0.50$.

In the nuclear matter within the mean field level, we can find that masses of $\bar{D}, \bar{D}^{*}, \bar{D}_{0}^{*}$ and $\bar{D}_{1}$ mesons are determined by

$$
m_{\bar{D}}^{*}=m-\frac{\Delta_{m} \sigma_{0}^{*}}{2 f_{\pi}}-\frac{\Delta_{\bar{D}}}{2}, m_{\bar{D}^{*}}^{*}=m-\frac{\Delta_{m} \sigma_{0}^{*}}{2 f_{\pi}}+\frac{\Delta_{\bar{D}^{*}}}{2}
$$




$$
m_{\bar{D}_{0}^{*}}^{*}=m+\frac{\Delta_{m} \sigma_{0}^{*}}{2 f_{\pi}}-\frac{\Delta_{\bar{D}_{0}^{*}}}{2}, m_{\bar{D}_{1}}^{*}=m+\frac{\Delta_{m} \sigma_{0}^{*}}{2 f_{\pi}}+\frac{\Delta_{\bar{D}_{1}}}{2}
$$

where the density dependent of $\sigma_{0}^{*}$ is determined by the gap equation (ㄹ.3). Note that in Eq. (‥6), we have added $\Delta_{\bar{D}}=202 \mathrm{MeV}, \Delta_{\bar{D}^{*}}=80 \mathrm{MeV}, \Delta_{\bar{D}_{0}^{*}}=164 \mathrm{MeV}$ and $\Delta_{\bar{D}_{1}}=54 \mathrm{MeV}$ in such a way that we can take into account the violation of heavy quark spin symmetry to make our study more realistic. In Sec. B, we compute the modifications of $\bar{D}$ mesons in the nuclear matter by one loop corrections as well as the mean field level.
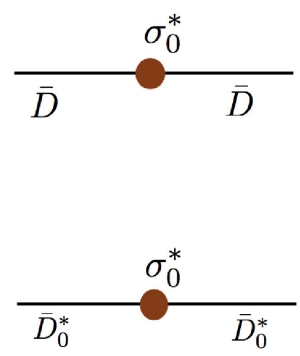
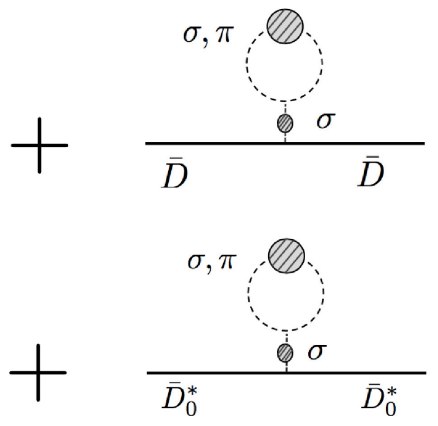
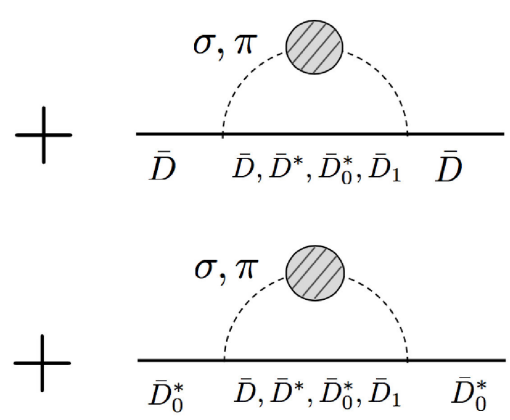

Figure 1: (color onlin) Mean field and one-loop corrections to the self-energy for $\bar{D}$ meson and $\bar{D}_{0}^{*}$ meson. Blobs indicate infinite sums of nucleon loops.

\section{Results and a summary}

In calculating the medium modifications to $\bar{D}$ meson and $\bar{D}_{0}^{*}$ meson in nuclear matter, we take into account the mean field $\sigma_{0}^{*}$ and one loop corrections in Fig. $\mathrm{W}$. In this figure, propagators of pion and $\sigma$ meson are given by the inverse of second functional derivative of the effective action in Eq. (2.2) with respect to $\pi^{a}$ and $\sigma$ on the background of $\sigma_{0}^{*}$, so that they include infinite sum of nucleon loops. Note that we can maintain the chiral symmetry by employing this procedure.

First, we show the density dependence of the masses of $\bar{D}$ and $\bar{D}_{0}^{*}$ mesons. The mass of $\bar{D}$ meson is defined by the solution of $q_{0}^{2}-m_{\bar{D}}^{* 2}-\operatorname{Re} \Sigma^{R}\left(q_{0}, \overrightarrow{0}\right)=0$, while mass of $\bar{D}$ meson is defined by the value of $q_{0}$ which realizes the maximum of peak of $\bar{D}_{0}^{*}$ resonance. The result is indicated in Fig. $\square$. Blue (red) circles show the mass of $\bar{D}\left(\bar{D}_{0}^{*}\right)$ meson at each densities. Filled circles are the results with all one-loop corrections. Open circles show the masses of $\bar{D}$ and $\bar{D}_{0}^{*}$ mesons with only mean field $\sigma_{0}^{*}$ plotted as a reference. The dashed lines refer the mass of $\bar{D}$ and $\bar{D}_{0}^{*}$ mesons in the vacuum. Mass of $\bar{D}$ increases while mass of $\bar{D}_{0}^{*}$ mesons decreases as the density increases, which reflects the partial restoration of chiral symmetry in the nuclear matter. Note that density dependence of masses of $\bar{D}$ and $\bar{D}_{0}^{*}$ mesons are mainly determined by the mean field $\sigma_{0}^{*}$, and one loop corrections are rather suppressed.

Second, we show the density dependence of the spectral function for $\bar{D}_{0}^{*}$ meson channel in Fig. [1]. Colored curve is the obtained result, and dashed black curve is the spectral function in the vacuum. Vertical dotted line refers the threshold of $\bar{D}+\pi$ of mean field level. The obtained result shows three peaks in the spectral function for $\bar{D}_{0}^{*}$ meson at density. First peak from right corresponds to the resonance of $\bar{D}_{0}^{*}$ meson state, whose peak position shifts to the lower energy as 


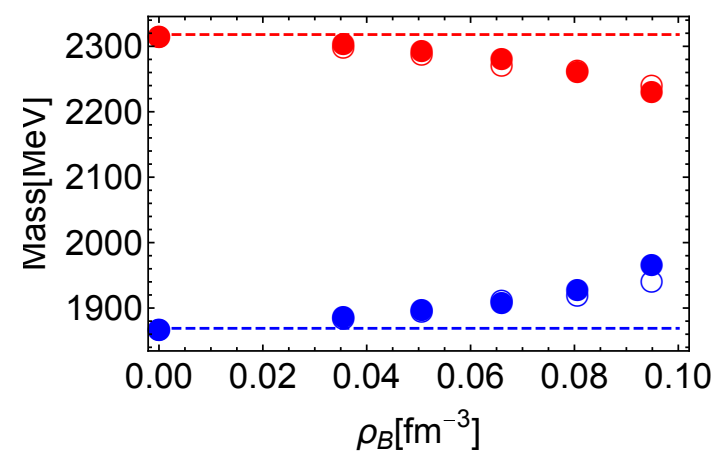

Figure 2: (color online) Density dependence of mass of $\bar{D}$ and $\bar{D}_{0}^{*}$.
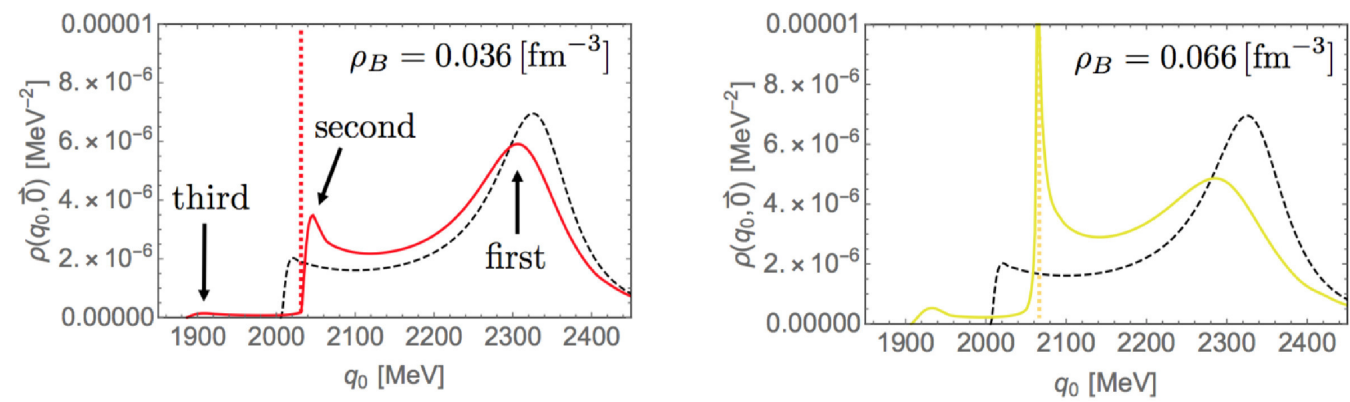

Figure 3: (color online) Spectral function for $\bar{D}_{0}^{*}$ meson channel at $\rho_{B}=0.036\left[\mathrm{fm}^{-3}\right]$ and $\rho_{B}=0.066$ $\left[\mathrm{fm}^{-3}\right]$. The detailed explanation is given in the text.

the density increases. The hight of this peak gets small and its width gets broadened. These changes are caused by the collisional broadening. Second peak from right is understood as the threshold enhancement. This enhancement is obtained since mass of $\bar{D}$ meson and $\bar{D}_{0}^{*}$ meson tends to get close. This peak is remarkably enhanced as the density increases, so that this peak can be a good probe to observe the chiral restoration in nuclear matter in future experiments. Third peak from right is identified as the Landau damping and this peak grows gradually as the density increases.

The modifications of mass of $\bar{D}$ meson and $\bar{D}_{0}^{*}$ meson in Fig. $\square$ and spectral function for $\bar{D}_{0}^{*}$ meson in Fig. [ ] reflect the tendency of partial restoration of chiral symmetry in nuclear matter. Therefore, we need future experiments to observe them to get clues to see the partial restoration of chiral symmetry in nuclear matter.

\section{References}

[1] T. Hatsuda and T. Kunihiro, Phys. Rept. 247, 221 (1994) doi:10.1016/0370-1573(94)90022-1 [hep-ph/9401310].

[2] D. Suenaga, S. Yasui and M. Harada, arXiv:1703.02762 [nucl-th].

[3] M. A. Nowak, M. Rho and I. Zahed, Phys. Rev. D 48, 4370 (1993) doi:10.1103/PhysRevD.48.4370 [hep-ph/9209272], W. A. Bardeen and C. T. Hill, Phys. Rev. D 49, 409 (1994). 